\title{
Modelling of errors due to speed of sound variations in photoacoustic tomography using a Bayesian framework
}

\author{
Jenni Tick ${ }^{1}$, Aki Pulkkinen ${ }^{1}$, and Tanja Tarvainen ${ }^{1,2}$ \\ ${ }^{1}$ Department of Applied Physics, University of Eastern Finland, P.O.Box 1627, \\ 70211 Kuopio, Finland \\ 2 Department of Computer Science, University College London, Gower Street, \\ London WC1E 6BT, United Kingdom \\ E-mail: jenni.tick@uef.fi
}

\begin{abstract}
Inverse problem of estimating initial pressure in photoacoustic tomography is ill-posed and thus sensitive to errors in modelling and measurements. In practical experiments, accurate knowledge of the speed of sound of the imaged target is commonly not available, and therefore an approximate speed of sound is used in the computational model. This can result in errors in the solution of the inverse problem that can appear as artefacts in the reconstructed images. In this paper, the inverse problem of photoacoustic tomography is approached in a Bayesian framework. Errors due to uncertainties in the speed of sound are modelled using Bayesian approximation error modelling. Estimation of the initial pressure distribution together with information on the reliability of these estimates are considered. The approach was studied using numerical simulations. The results show that uncertainties in the speed of sound can cause significant errors in the solution of the inverse problem. However, modelling of these uncertainties improves the accuracy of the solution.
\end{abstract}

Keywords: photoacoustic tomography, inverse problems, Bayesian methods, modelling of errors, uncertainty quantification 


\section{Introduction}

Photoacoustic tomography (PAT) is a hybrid imaging technique that combines strong optical contrast and high ultrasonic resolution in a single modality. It has a variety of applications in biomedical imaging $[1,2,3,4,5,6,7]$. In PAT measurement situation, absorption of an externally illuminated short light pulse induces a transient pressure distribution inside the medium through a photoacoustic effect. This pressure propagates through the medium as an acoustic wave and can be measured on the boundary of the target using ultrasound sensors. Then, in the image reconstruction problem of PAT, the initial pressure is reconstructed from the measured ultrasound signal. As an alternative to the conventional image reconstruction, a full solution of the inverse problem, i.e. posterior probability density, can be examined [8, 9].

In many inverse problems methodologies used in PAT, the numerical solution of the forward model is required in the solution of the inverse problem. These methods require knowledge of the speed of sound. However, in practical experiments, the speed of sound is usually not known accurately. In fact, in many of the PAT reconstruction schemes, the speed of sound is considered as a known (fixed, often constant) nuisance parameter. This can cause severe errors in the solution of the inverse problem. Therefore, various methods have been proposed to mitigate the issue. For example, a constant speed of sound has been optimized according to some image quality metric, such as image sharpness [10]. However, such approach can be difficult to apply when the actual speed of sound distribution is not constant. In addition, repeating image reconstruction with several speed of sound values can become time consuming. Alternatively, in the halftime and partial-time image reconstruction methods, an image is reconstructed from a dataset that has been temporally truncated to exclude the data components that have been strongly aberrated $[11,12]$. Although this approach reduces artefacts induced by acoustic heterogeneities, significant distortions can remain, especially in cases where broad range of acoustic properties is present. In addition, temporal truncation leads to loss of data that can cause problems by increasing the ill-posedness of the problem. On the other hand, small speed of sound variations have also been compensated by estimating the acoustic travel time using correlation information between measured signals [13]. Correspondingly, artefacts induced by strong acoustic heterogeneities have been mitigated by weighting the back-projected signals by the probability that the signal is being undisturbed during the propagation [14].

Basically, the speed of sound could be jointly estimated with the initial pressure distribution [15, 16, 17, 18, 19]. However, this is generally an unstable problem [20]. Stabilization of the problem has been proposed by reducing the number of unknowns for example by utilizing information of the structure of the speed of sound distribution [19]. Alternatively, adjunct imaging data, such as ultrasound tomography or passive-element measurements, can be utilized to estimate the speed of sound distribution [21, 22, 23, 24]. Unfortunately, not all PAT measurement devices can conduct these measurements. In addition, an integration of these adjunct imaging modalities with PAT measurement 
setups can be challenging.

In this work, the inverse problem of PAT is approached in a Bayesian framework to ill-posed inverse problems [25, 26, 8]. Therefore, all parameters are treated as random variables that are characterized by their probability distributions. Measurements, model of the imaging situation and prior information are used to infer the probability distributions of the unknown parameters of primary interest. That is, we solve the conditional distribution of the initial pressure in each pixel of the discretized domain, i.e. the full posterior distribution. In addition, point estimates for images of the unknown initial pressure and its credibility are inspected. The Bayesian framework facilitates representing and taking into account uncertainties in parameters, models, and geometries $[27,28,29,30,31,32,33,34,35,36,37]$. In this work, modelling of errors due to speed of sound variations is studied by Bayesian approximation error modelling [25]. In the approach, these errors are approximated as Gaussian distributed noise and included into the noise model of the data likelihood.

The paper is organized as follows. Section 2 gives a brief review of the forward model and the inverse problem of PAT in the Bayesian framework. In addition, the Bayesian approximation error approach for modelling errors due to unknown speed of sound in PAT is presented. In Section 3, approach is evaluated with simulations. Finally, discussions and conclusions are given in Sections 4 and 5.

\section{Photoacoustic tomography}

\subsection{Forward model}

The forward problem in PAT is to solve the acoustic field caused by absorption of a light pulse as a function of time at the sensors surrounding the object when the acoustic properties of the medium are known. The acoustic wave propagation in a non-absorbing medium and free space can be described by a wave equation

$$
\left\{\begin{array}{l}
\left(\frac{\partial^{2}}{\partial t^{2}}-c(r)^{2} \nabla^{2}\right) p(r, t)=0, \quad r \in \mathbb{R}^{d}, t \in[0, T[ \\
p(r, t=0)=p_{0}(r), \quad \text { when } r \in \Omega \subset \mathbb{R}^{d}, 0 \text { otherwise } \\
\frac{\partial}{\partial t} p(r, t=0)=0
\end{array}\right.
$$

where $p(r, t)$ is the acoustic pressure at point $r$ and time $t, p_{0}(r)$ is the initial pressure distribution, and $c(r)$ is the speed of sound $[6,2]$. In PAT measurements, the acoustic pressure wave $p(r, t)$ is only recorded over a subset $S$ of the boundary of the target $\Omega$ for some time $t=0$ to $T$, with $\Omega$ being an imaging domain, and $T$ being the duration of time that the photoacoustic time series is captured for. In this paper, the solution of the wave equation is numerically approximated using a $k$-space time-domain method implemented with the $\mathrm{k}$-Wave MATLAB toolbox [38]. 


\subsection{Inverse problem}

The inverse problem of PAT is to estimate the initial pressure distribution given the measured pressure waves based on an observation model. In this paper, a discrete observation model for PAT in the presence of an additive noise is considered which can be written as

$$
p_{t}=K(c) p_{0}+e
$$

where $p_{t} \in \mathbb{R}^{m}$ is a vector composed of the acoustic pressure waves sampled at discrete set of sensors at discrete time points, $p_{0} \in \mathbb{R}^{n}$ is the discrete initial pressure distribution, $K(c) \in \mathbb{R}^{m \times n}$ is the linear operator which maps the initial pressure distribution to the measured data by discretizing the forward model (1), and $e \in \mathbb{R}^{m}$ denotes the noise. In this paper, the matrix $K(c)$ is formed by utilizing the k-Wave toolbox by looping over each pixel describing $p_{0}$, setting the pixel value to one while keeping the other pixels at zero and computing the acoustic output [8]. These outputs then form the columns of the matrix $K(c)$. This corresponds to computing the impulse response of a discrete system approximating (1).

In this paper, the solution of the inverse problem is based on the Bayesian approach $[26,25]$. Let us consider all parameters as random variables. The solution of the inverse problem is the posterior density $\pi\left(p_{0} \mid p_{t}\right)$, and it can be written using the Bayes' formula as

$$
\pi\left(p_{0} \mid p_{t}\right) \propto \pi\left(p_{0}\right) \pi\left(p_{t} \mid p_{0}\right),
$$

where $\pi\left(p_{0}\right)$ is the prior probability density and $\pi\left(p_{t} \mid p_{0}\right)$ is the likelihood density.

The likelihood density can be written as

$$
\pi\left(p_{t} \mid p_{0}\right)=\int \pi\left(p_{t}, e \mid p_{0}\right) \mathrm{d} e=\int \pi\left(p_{t} \mid p_{0}, e\right) \pi\left(e \mid p_{0}\right) \mathrm{d} e .
$$

Given the observation model (2), we have $\pi\left(p_{t} \mid p_{0}, e\right)=\delta\left(p_{t}-K(c) p_{0}-e\right)$ and the likelihood becomes

$$
\pi\left(p_{t} \mid p_{0}\right)=\int \delta\left(p_{t}-K(c) p_{0}-e\right) \pi\left(e \mid p_{0}\right) \mathrm{d} e=\pi_{e \mid p_{0}}\left(p_{t}-K(c) p_{0} \mid p_{0}\right) .
$$

In the case $p_{0}$ and $e$ are mutually independent

$$
\pi\left(p_{t} \mid p_{0}\right)=\pi_{e}\left(p_{t}-K(c) p_{0}\right),
$$

where $\pi_{e}$ is the probability density of the noise $e$. This leads to a posterior distribution

$$
\pi\left(p_{0} \mid p_{t}\right) \propto \pi\left(p_{0}\right) \pi_{e}\left(p_{t}-K(c) p_{0}\right) .
$$

That is, in the above, noise $e$ was premarginalized and is not present in (7).

In this paper, a Gaussian distributed prior $p_{0} \sim \mathcal{N}\left(\eta_{p_{0}}, \Gamma_{p_{0}}\right)$ with mean $\eta_{p_{0}}$ and covariance matrix $\Gamma_{p_{0}}$ is considered. In addition, noise is assumed a Gaussian i.e. $e \sim \mathcal{N}\left(\eta_{e}, \Gamma_{e}\right)$, where $\eta_{e}$ is the mean and $\Gamma_{e}$ is the covariance matrix. Thus, the posterior density (7) is also a Gaussian distribution $p_{0} \mid p_{t} \sim \mathcal{N}\left(\eta_{p_{0} \mid p_{t}}, \Gamma_{p_{0} \mid p_{t}}\right)$, where

$$
\eta_{p_{0} \mid p_{t}}=\Gamma_{p_{0} \mid p_{t}}\left(K(c)^{\mathrm{T}} \Gamma_{e}^{-1}\left(p_{t}-\eta_{e}\right)+\Gamma_{p_{0}}^{-1} \eta_{p_{0}}\right)
$$




$$
\Gamma_{p_{0} \mid p_{t}}=\left(K(c)^{\mathrm{T}} \Gamma_{e}^{-1} K(c)+\Gamma_{p_{0}}^{-1}\right)^{-1}
$$

are the mean and covariance of the posterior distribution, respectively.

In this paper, the entire posterior density is determined and inspected. The mean of the posterior, which corresponds to the maximum a posteriori estimate, is visualized to inspect the estimated initial pressure as an image. Furthermore, reliability of the estimates is assessed by determining pixel-wise standard deviations. In addition, marginal densities of the posterior distribution are computed $p_{0, k} \mid p_{t} \sim$ $\mathcal{N}\left(\eta_{p_{0} \mid p_{t}, k}, \Gamma_{p_{0} \mid p_{t}, k k}\right)$, where $\eta_{p_{0} \mid p_{t}, k}$ is the value of $\eta_{p_{0} \mid p_{t}}$ in the $k$ th pixel and $\Gamma_{p_{0} \mid p_{t}, k k}$ is the value of the $k$ th diagonal element of $\Gamma_{p_{0} \mid p_{t}}$. Covariances of pixels are also inspected.

\subsection{Inverse problem in the presence of modelling errors}

The inverse problem may be simplified by using an approximate model. The approximate model can be, for example an approximation of a complex physical model, a discrete model with discretization errors larger than measurement precision, or model with uncertainties such as unknown boundary shape. In this paper, we study a situation in which the exact speed of sound is not known and instead we have approximate knowledge of it. We denote the corresponding forward model as $K(\widetilde{c})$ where $\widetilde{c}$ is a constant or piece-wise constant (nominal) value for the speed of sound.

If an approximate forward model $K(\widetilde{c})$ is utilized, the observation model can be written utilizing the Bayesian approximation error modelling [25] in the form

$$
\begin{aligned}
p_{t} & =K(\widetilde{c}) p_{0}+\epsilon+e \\
& =K(\widetilde{c}) p_{0}+n,
\end{aligned}
$$

where $n=\epsilon+e$ is total error, and $\epsilon=K(c) p_{0}-K(\widetilde{c}) p_{0}$ is approximation error describing the modelling error between the exact and the approximate model.

Now, observation model (10) is used and premarginalization over the measurement noise $e$ and modelling error $\epsilon$ is carried out by following the approach in [39]. Thus, the likelihood is

$$
\pi\left(p_{t} \mid p_{0}\right)=\int \pi\left(p_{t}, n \mid p_{0}\right) \mathrm{d} n
$$

With the help of the joint density

$$
\begin{aligned}
\pi\left(p_{t}, n, p_{0}\right) & =\pi\left(p_{t} \mid n, p_{0}\right) \pi\left(n \mid p_{0}\right) \pi\left(p_{0}\right) \\
& =\pi\left(p_{t}, n \mid p_{0}\right) \pi\left(p_{0}\right)
\end{aligned}
$$

the likelihood can be written as

$$
\pi\left(p_{t} \mid p_{0}\right)=\int \pi\left(p_{t} \mid n, p_{0}\right) \pi\left(n \mid p_{0}\right) \mathrm{d} n
$$

Given the observation model (10), we have $\pi\left(p_{t} \mid n, p_{0}\right)=\delta\left(p_{t}-K(\widetilde{c}) p_{0}-n\right)$ yielding the likelihood

$$
\begin{aligned}
\pi\left(p_{t} \mid p_{0}\right) & =\int \delta\left(p_{t}-K(\widetilde{c}) p_{0}-n\right) \pi\left(n \mid p_{0}\right) \mathrm{d} n \\
& =\pi_{n \mid p_{0}}\left(p_{t}-K(\widetilde{c}) p_{0} \mid p_{0}\right)
\end{aligned}
$$


If we ignore the mutual dependence of $n$ and $p_{0}$,

$$
\pi\left(p_{t} \mid p_{0}\right)=\pi_{n}\left(p_{t}-K(\widetilde{c}) p_{0}\right) .
$$

Let us assume that noise is Gaussian distributed $e \sim \mathcal{N}\left(\eta_{e}, \Gamma_{e}\right)$, and approximate the modelling error as Gaussian $\epsilon \sim \mathcal{N}\left(\eta_{\epsilon}, \Gamma_{\epsilon}\right)$. Thus, the total error is Gaussian distribution $n \sim \mathcal{N}\left(\eta_{n}, \Gamma_{n}\right)$ where $\eta_{n}=\eta_{e}+\eta_{\epsilon}$ and $\Gamma_{n}=\Gamma_{e}+\Gamma_{\epsilon}$, and a Gaussian approximation for the likelihood is obtained.

With a Gaussian prior, the posterior density is Gaussian $p_{0} \mid p_{t} \sim \mathcal{N}\left(\eta_{p_{0} \mid p_{t}}, \Gamma_{p_{0} \mid p_{t}}\right)$, where

$$
\begin{aligned}
\eta_{p_{0} \mid p_{t}} & =\Gamma_{p_{0} \mid p_{t}}\left(K(\widetilde{c})^{\mathrm{T}} \Gamma_{n}^{-1}\left(p_{t}-\eta_{n}\right)+\Gamma_{p_{0}}^{-1} \eta_{p_{0}}\right) \\
\Gamma_{p_{0} \mid p_{t}} & =\left(K(\widetilde{c})^{\mathrm{T}} \Gamma_{n}^{-1} K(\widetilde{c})+\Gamma_{p_{0}}^{-1}\right)^{-1} .
\end{aligned}
$$

It should be noted that as $K(\widetilde{c}) \rightarrow K(c)$ the estimates approach each other.

\section{Numerical studies}

In the simulations, two problems were considered. In the first problem, a numerical soft tissue mimicking phantom that possessed a spatially variable speed of sound was studied. In the second problem, a numerical soft tissue and bone mimicking phantom that consisted of two regions with varying speed of sounds was examined. In this problem, boundaries of the regions were assumed to be known. In practice, this kind of information could be received for example from computed tomography image.

The inverse problem was solved using three different likelihood models: 1) an accurate forward model (AFM) using $K(c)$ that presents (unrealistic) situation where the speed of sound is known exactly, 2) an inexact forward model (IFM) with $K(\widetilde{c})$ that represents typical situation where some preassigned values for the speed of sound are used, and 3) an inexact forward model with error modelling (IFM\&EM). In the case of the AFM, the posterior density was solved using (8)-(9). In the case of the IFM, (16)-(17) were used. If modelling errors were ignored, we set the modelling error as $\epsilon=0$ resulting in $n \sim \mathcal{N}\left(\eta_{e}, \Gamma_{e}\right)$.

For IFM\&EM, modelling error was computed by sampling and approximating it as a Gaussian as follows. First, a set of samples $\left\{p_{0}^{(l)}, l=1, \ldots, N_{s}\right\}$ were drawn from the teaching distribution of the initial pressure. In addition, a set of samples $\left\{c^{(l)}, l=1, \ldots, N_{s}\right\}$ were drawn from the teaching distribution of the speed of sound. Then, samples of the approximation error were computed using

$$
\epsilon^{(l)}=K\left(c^{(l)}\right) p_{0}^{(l)}-K(\widetilde{c}) p_{0}^{(l)}
$$

where a constant or piece-wise constant speed of sound was used in the inexact forward model $K(\widetilde{c})$. Further, the mean and covariance of the approximation error were computed as

$$
\eta_{\epsilon}=\frac{1}{N_{s}} \sum_{l=1}^{N_{s}} \epsilon^{(l)}
$$



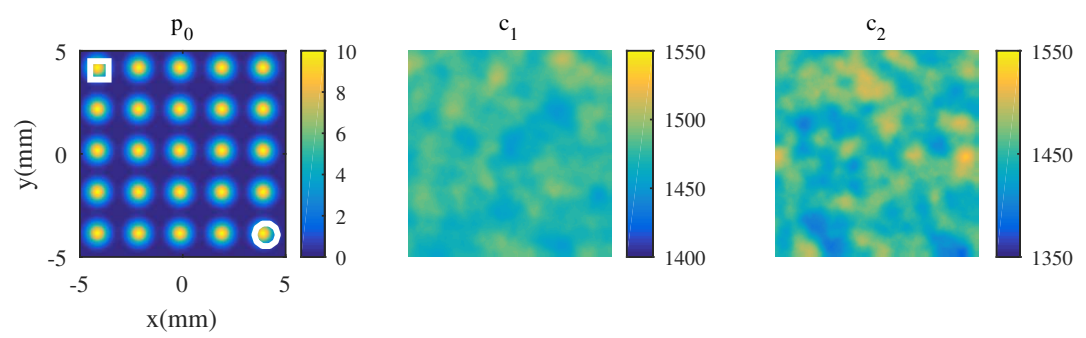

Figure 1. The simulated (true) initial pressure distribution $p_{0}$ and the speed of sound distributions $c_{1}$ and $c_{2}$ used in the soft tissue mimicking simulations. The initial pressure distribution is given in arbitrary units and the speed of sound distributions are given in units of $\mathrm{m} / \mathrm{s}$. The square and circle in the left image indicate the pixels where the covariances and marginal densities are plotted.

$$
\Gamma_{\epsilon}=\frac{1}{N_{s}-1} \sum_{l=1}^{N_{s}}\left(\epsilon^{(l)}-\eta_{\epsilon}\right)\left(\epsilon^{(l)}-\eta_{\epsilon}\right)^{\mathrm{T}} .
$$

It should be noted that simulation of the approximation error statistics is a computationally intensive task, since the computation time for setting up the error model is roughly equivalent to two times the number of samples multiplied by the time for forward solution. However, this can be done off-line and the error model needs to be estimated only once for a fixed measurement setup, and for the expected range of uncertainties.

The accuracy of the posterior mean estimates was evaluated by computing the relative errors of the estimates with respect to the true initial pressure distribution using

$$
E_{p_{0}}=100 \% \cdot \frac{\left\|p_{0}-\hat{p}_{0}\right\|}{\left\|p_{0}\right\|},
$$

where $p_{0}$ is the simulated initial pressure distribution and $\hat{p}_{0}$ is the estimated value interpolated to the simulation space.

\subsection{Soft tissue mimicking simulations}

3.1.1. Data simulation In the simulations, a square domain of size $10 \mathrm{~mm} \times 10 \mathrm{~mm}$ was considered. The sensors were placed in one full view and two limited view sensor geometries. The full view (four side) configuration contained 100 sensors surrounding the domain. In the first limited view geometry, 50 sensors were placed in two adjacent sides of the domain (two side), and in the other geometry, 25 sensors were placed in one side of the domain (one side).

In data simulation, the medium was modelled as inhomogeneous. The simulated initial pressure distribution consisted of a homogeneous background with initial pressure zero that included 25 Gaussian inclusions that had a peak value of 10 and a full-width at half-maximum of $0.8 \mathrm{~mm}$. The inclusions were located in a $5 \times 5$ grid pattern. The simulated initial pressure distribution is illustrated in Figure 1. Two different speed 
Table 1. Parameters of the squared exponential distribution used in generating the speed of sound distributions and in teaching the modelling error in the soft tissue mimicking simulations: mean $\eta_{c}(\mathrm{~m} / \mathrm{s})$, standard deviation $\sigma_{c}(\mathrm{~m} / \mathrm{s})$ and characteristic length scale $l_{c}(\mathrm{~mm})$.

\begin{tabular}{lcccccccc}
\hline & \multicolumn{4}{c}{$c_{1}$} & & \multicolumn{3}{c}{$c_{2}$} \\
\cline { 2 - 4 } \cline { 6 - 8 } & $\eta_{c}$ & $\sigma_{c}$ & $l_{c}$ & & $\eta_{c}$ & $\sigma_{c}$ & $l_{c}$ \\
\hline Data simulation & 1475 & $50 / 6$ & 0.75 & & 1450 & $125 / 6$ & 0.75 \\
Modelling error & 1470 & $100 / 6$ & 1 & & 1470 & $100 / 6$ & 1 \\
\hline
\end{tabular}

of sound distributions $c_{1}$ and $c_{2}$ were considered and they are shown in Figure 1. The speed of sound distributions were chosen to imitate a heterogeneous soft tissue. The generated speed of sound distributions $c_{1}$ and $c_{2}$ were drawn from a squared exponential distribution [40] using parameters which are shown in Table 1. The speed of sound distribution $c_{1}$ was generated from distribution with $99.7 \%$ in the range of $[1450,1500] \mathrm{m} / \mathrm{s}$ and $c_{2}$ from broader distribution in range of $[1388,1513] \mathrm{m} / \mathrm{s}$.

Photoacoustic data was generated using the k-Wave MATLAB toolbox [38]. The simulation grid consisted of $300 \times 300$ pixels with $33 \mu \mathrm{m}$ pixel width. The pressure signals were recorded for 2401 time steps at a temporal sampling rate of $200 \mathrm{MHz}$ to ensure the numerical stability of the forward model. However, the grid used in the solution of the inverse problem supports only much lower frequencies. Thus, the simulated signal was downsampled to $33 \mathrm{MHz}$. A Gaussian noise with a standard deviation of $1 \%$ of the maximum value of the simulated data was added to the simulated pressure signals.

3.1.2. Approximation of the modelling errors In order to form an approximation of the modelling error, 10000 samples were drawn from the teaching distributions of the initial pressure and speed of sound. The teaching distribution for the initial pressure was the Ornstein-Uhlenbeck process [40] with parameters listed in Table 2. In case that negative parameter values were drawn, absolute values of them were taken in order to make the samples positive and thus keep the model physical. For the speed of sound, a squared exponential distribution with parameters given in Table 1 was used as the teaching distribution. With these parameters, $99.7 \%$ of the speed of sound values were expected to be normally distributed within the range $[1420,1520] \mathrm{m} / \mathrm{s}$. For the inexact forward model, a constant speed of sound value $1500 \mathrm{~m} / \mathrm{s}$ was used. Then, samples of the approximation error were computed from a set of forward solutions using (18) and the mean and covariance of the modelling error were approximated from these samples using (19) and (20).

3.1.3. Solution of the inverse problem For the solution of the inverse problem, the computation domain was discretized into $200 \times 200$ pixels with $50 \mu \mathrm{m}$ pixel width. In the case of the AFM, the true speed of sound distribution interpolated from the simulation 
Table 2. Parameters of the Ornstein-Uhlenbeck distribution used in simulating the initial pressure distributions when teaching the modelling error and in the solution of the inverse problem in the case of the soft tissue mimicking simulations: mean $\eta_{p_{0}}$, standard deviation $\sigma_{p_{0}}$ and characteristic length scale $l_{p_{0}}(\mathrm{~mm})$.

\begin{tabular}{cccc}
\hline & $\eta_{p_{0}}$ & $\sigma_{p_{0}}$ & $l_{p_{0}}$ \\
\hline Modelling error & 5 & $10 / 6$ & 0.75 \\
Inverse problem & 5 & $10 / 2$ & 0.75 \\
\hline
\end{tabular}

domain to the reconstruction domain was used. In the case of the IFM, fixed constant value for the speed of sound of $1500 \mathrm{~m} / \mathrm{s}$ was used.

The Ornstein-Uhlenbeck process was used as the prior model for the initial pressure. The parameters of the prior model are given in Table 2. For the measurement noise, a Gaussian distributed noise with zero mean and standard deviation set to $1 \%$ of the peak positive amplitude of the noisy simulated data was used. Furthermore, in the case of the IFM, two situations were considered: ignoring the modelling errors (IFM) and utilizing the simulated modelling error distribution (IFM\&EM).

3.1.4. Results The mean and standard deviation of the posterior distribution of the initial pressure obtained using different likelihood models and sensor geometries are shown in Figures 2 and 3 for the two different speed of sound distributions $c_{1}$ and $c_{2}$, respectively. Furthermore, marginal densities at two pixels inside of the domain are shown in Figure 4. The relative errors of the estimates are given in Table 3.

As it can be seen, the most accurate estimates for the initial pressure are obtained when the speed of sound is accurately known. In this case, also the relative errors are smallest. Using a constant value for the speed of sound causes errors into the posterior distribution that appear as artefacts in the images of the posterior mean which is especially evident in the two side sensor geometry. These artefacts cause significant increase in the relative errors. However, standard deviations are almost the same size as in the case of the exactly known speed of sound. This indicates that reliability of these estimates may not be realistic in the case of the inaccurate speed of sound. The errors in the estimated initial pressure can be reduced, if the uncertainties in the speed of sound are modelled and taken into account in the solution of the inverse problem. However, modelling of these errors does not compensate for all the errors in the posterior mean and some artefacts remain in the images, see for example the lower right corner of the posterior mean obtained using IFM\&EM and two side sensor geometry in Figures 2 and 3. This is reflected to relative errors that are lower than in the case of the constant speed of sound but slightly higher than the relative errors with the accurate speed of sound. On the other hand, modelling of the errors increases the width of the posterior distribution that can be seen in the standard deviations in Figures 2 and 3 and especially in the marginal densities in Figure 4. That is, although all the errors cannot be reduced, 

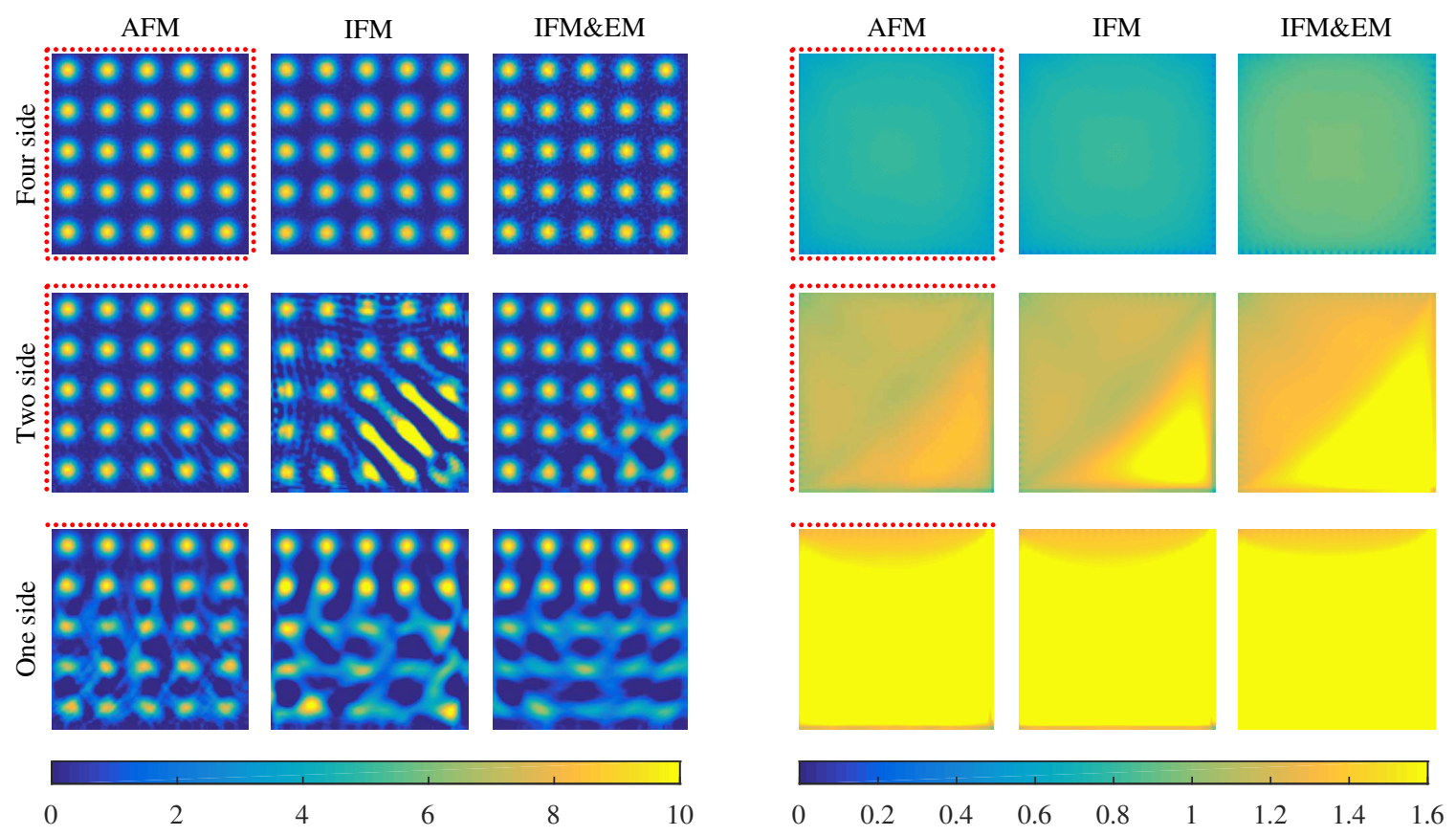

Figure 2. Posterior mean (left block) and standard deviation (right block) for the soft tissue mimicking simulations with the speed of sound distribution $c_{1}$. Shown in the blocks are the results obtained using the AFM, IFM and IFM\&EM. The rows from top to bottom represent the four side (first row), two side (second row) and one side (third row) sensor geometries. The red dots in the first column images indicate the locations of the sensors.

Table 3. Relative errors of the estimated mean of the posterior in percentage obtained using the AFM, IFM and constant IFM\&EM in four side, two side and one side sensor geometries in the case of the soft tissue mimicking simulations.

\begin{tabular}{cccccccc}
\hline & \multicolumn{3}{c}{$c_{1}$} & & \multicolumn{3}{c}{$c_{2}$} \\
\cline { 2 - 4 } \cline { 6 - 8 } & AFM & IFM & IFM\&EM & & AFM & IFM & IFM\&EM \\
\hline Four side & 5 & 17 & 11 & & 3 & 33 & 20 \\
Two side & 8 & 122 & 28 & & 9 & 275 & 51 \\
One side & 31 & 74 & 55 & & 24 & 108 & 68 \\
\hline
\end{tabular}

the uncertainty of the estimates is increased, which indicates that the reliability of these estimates can be evaluated with better accuracy.

When comparing the different sensor geometries, it can be seen that the estimates of the posterior mean obtained using the full view sensor geometry are qualitatively and quantitatively better and the standard deviations are smaller than in the limited view geometries. As the sensor geometry turns more limited view, accuracy of the estimates is reduced and the estimates of the initial pressure become distorted in the areas not closed by the sensors. In addition, the standard deviations increase. This demonstrates behavior of inverse problems. That is, in the case of mildly ill-posed inverse problem, 

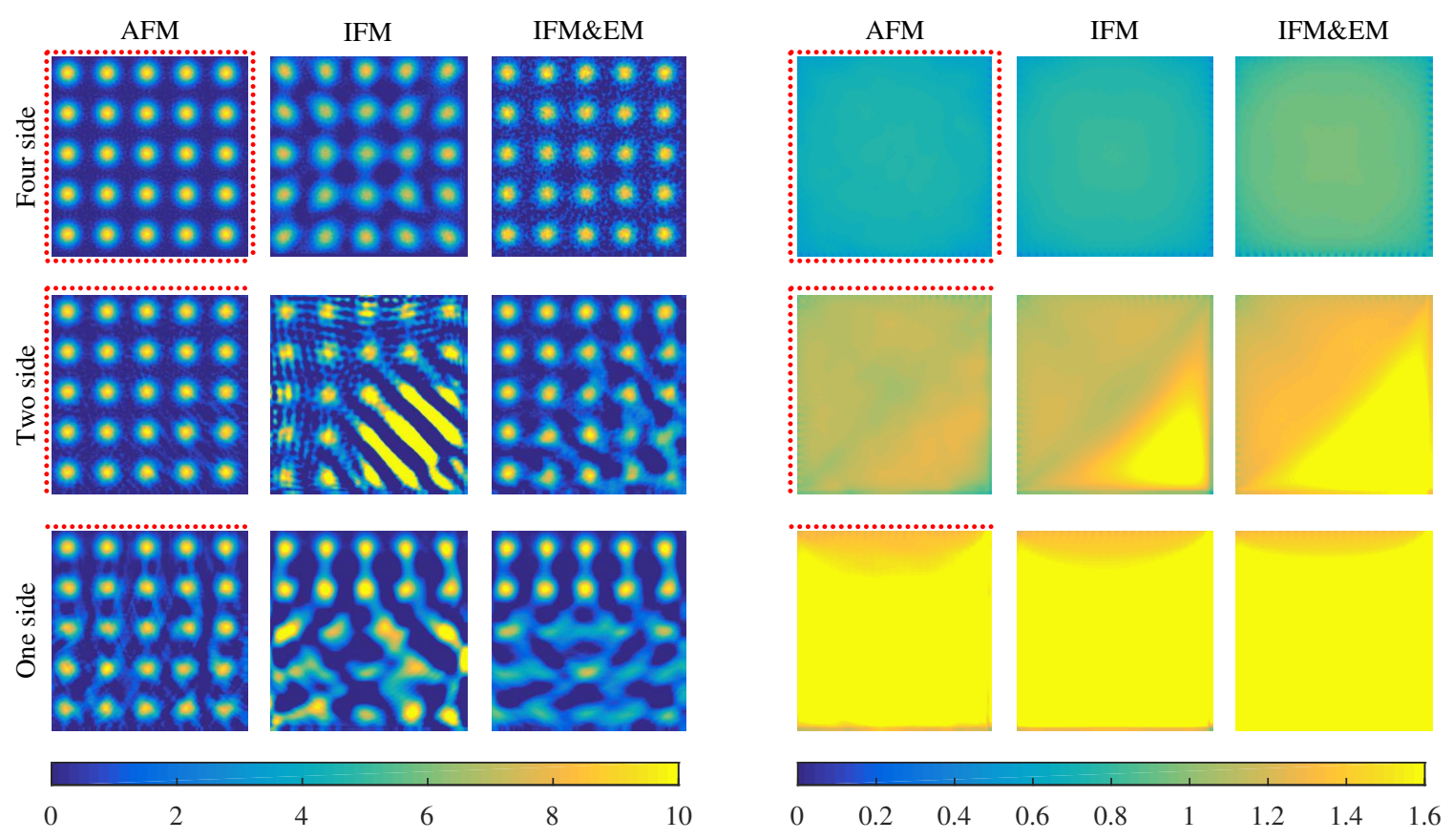

Figure 3. Posterior mean (left block) and standard deviation (right block) for the soft tissue mimicking simulations with the speed of sound distribution $c_{2}$. Shown in the blocks are the results obtained using the AFM, IFM and IFM\&EM. The rows from top to bottom represent the four side (first row), two side (second row) and one side (third row) sensor geometries. The red dots in the first column images indicate the locations of the sensors.

such as full-view measurement geometry and inaccuracies in the speed of sound, small errors may not cause large errors in the solution of the inverse problem. Further, in the case of ill-posed inverse problem, such as limited view measurement geometry, even small errors in modelling can cause large errors into the solution. However, it should also be noted that, although in the case of mildly ill-posed inverse problem, the estimated mean of the posterior distribution may appear qualitatively good and the relative error is relatively small, the width of the posterior may still be too narrow to be considered as a credible reliability estimate. This can be seen for example in the images on the first row of Figure 4, where marginal densities of the posterior distribution are narrow and thus the true values are not within their support in the cases where incorrect speed of sound value is used and its errors are not modelled.

If we compare the results of different speed of sound distributions, $c_{1}$ and $c_{2}$, it can be seen that even small increase in the variation of the speed of sound values in the simulated data, increases the errors in the estimates. Furthermore, it can be noticed that, although modelling of the errors was taught using speed of sound values with distribution closer to the wider distribution, it still cannot compensate the errors as well as in the case of the narrow distribution as can be seen from the images of posterior mean. This is due to fact that, in the case of PAT, even small variations in the speed of sound, can cause large variations in the measured time dependent photoacoustic wave. 

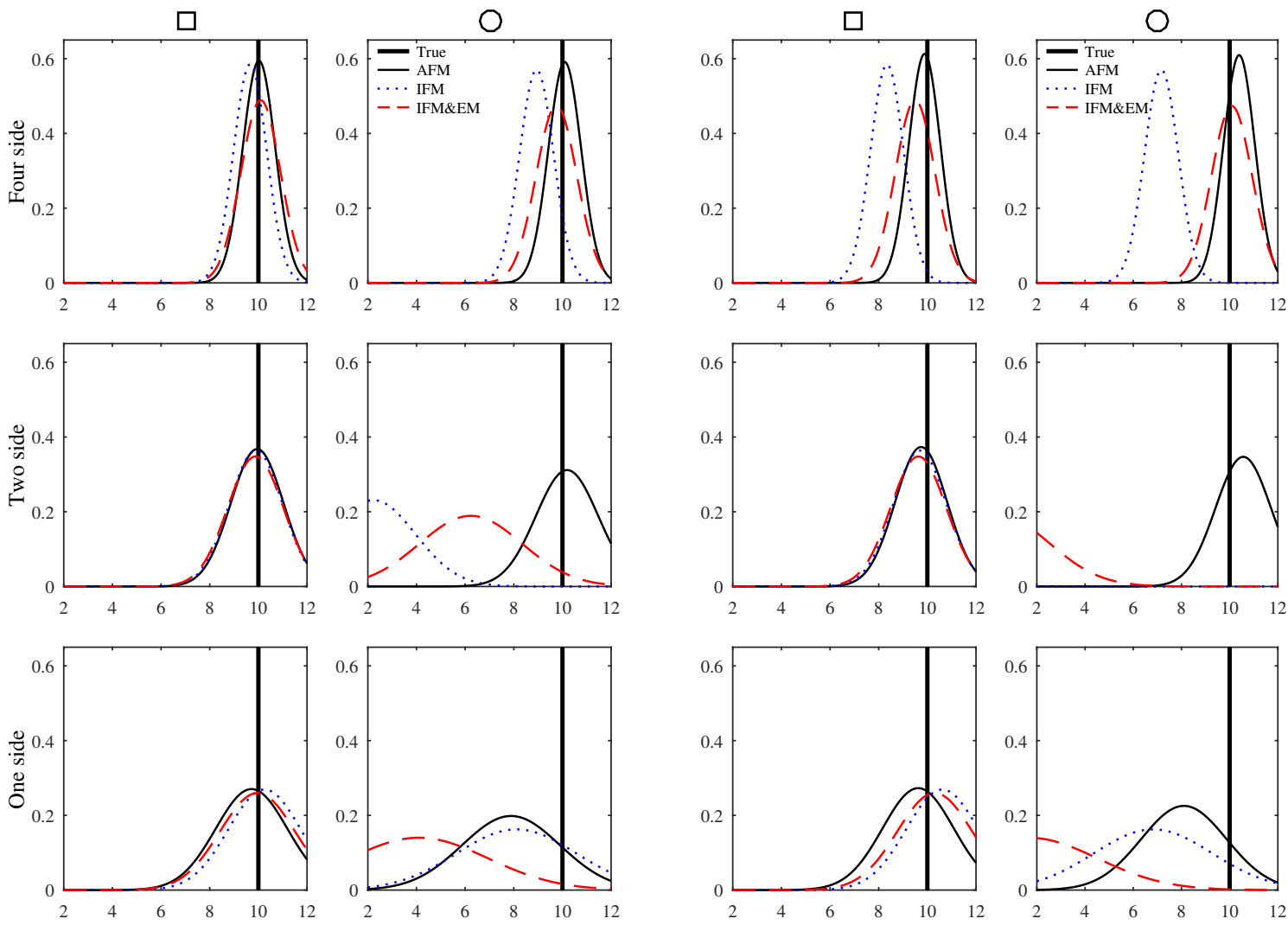

Figure 4. Marginal probability densities of the posterior distributions at locations denoted by $\square$ and $\bigcirc$ in Figure 1 for the soft tissue mimicking simulations. The left block shows results for the simulated speed of sound $c_{1}$ and the right block shows the results for $c_{2}$. The rows presents results obtained using four side (first row), two side (second row) and one side (third row) sensor geometries. Shown in the graphs are results for the AFM, IFM and IFM\&EM with black line denoting the true value initial pressure distribution.

Thus, the best result in compensation of errors would be achieved if the width of the teaching distribution is chosen judiciously and case-specifically.

To visualize covariance of the posterior distribution, covariance at two pixels inside of the domain were plotted and are shown in Figure 5 for the speed of sound distribution $c_{1}$. As it can be seen, both ignorance of modelling errors and modelling of these errors changes the covariance. Furthermore, it can be noticed that the covariance obtained using the full view sensor geometry has similar correlation to all directions. As sensor geometry turns more limited view, the correlation between the pixels increases and the shape of the correlation pattern changes. In addition, the point $(\bigcirc)$ that is far from the sensor correlates more strongly with other pixels than the point $(\square)$ that is close to the sensor. Furthermore, in the distant point, the correlation seems to have same direction as the limited view stripe artefacts.

\subsection{Soft tissue and bone mimicking simulations}



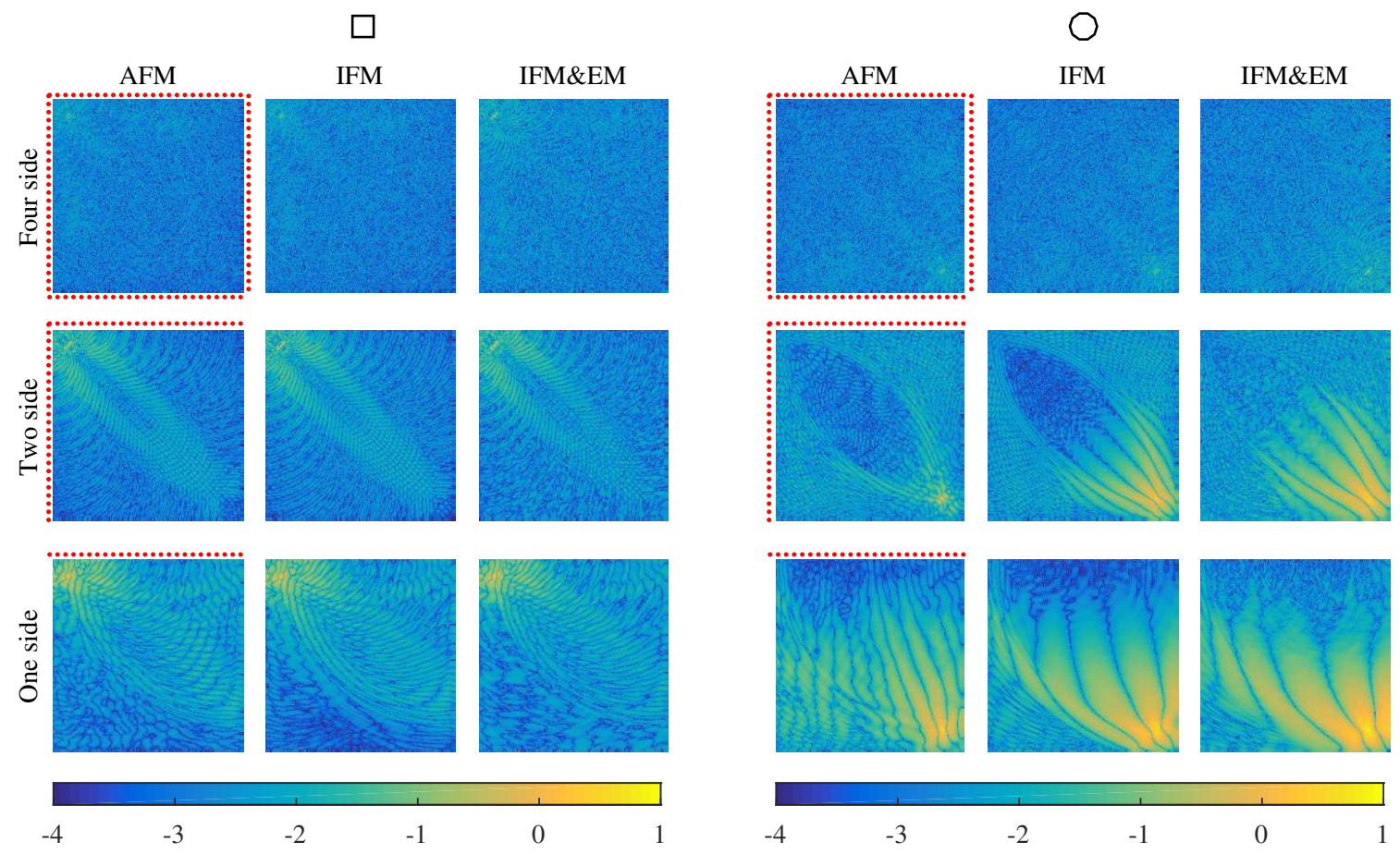

Figure 5. The absolute values of the posterior covariances of the initial pressure on a logarithmic scale at locations denoted by $\square$ and $\bigcirc$ in Figure 1 for the soft tissue mimicking simulations with the speed of sound distribution $c_{1}$. The left block presents the results in the point marked with $\square$ and the right block presents the results in the point marked with $\bigcirc$. Shown in the blocks are the results obtained using the AFM, IFM and IFM\&EM in four side (first row), two side (second row) and one side (third row) sensor geometry. The red dots in the first column images indicate the locations of the sensors.

3.2.1. Data simulation A more complicated situation in which the domain was composed of two sub-regions in which the speed of sound was significantly different was studied. The purpose of the simulation was to mimic the speed of sound in soft tissue and bone. The simulations were performed again in a square domain of size $10 \mathrm{~mm} \times 10 \mathrm{~mm}$. Same sensor geometries as in the previous simulations were used.

The initial pressure distribution and the speed of sound distribution employed to simulate photoacoustic data are shown in Figure 6. The initial pressure distribution consisted of many different shaped and sized somewhat smooth inclusions. The acoustic heterogeneity imitated a bony structure inside a soft tissue with shape of semicircle and thickness of $0.7 \mathrm{~mm}$. The speed of sound distribution was created using the squared exponential prior with parameters listed in Table 4, i.e. the speed of sound in the bony structure are on the interval $[2675,2845] \mathrm{m} / \mathrm{s}$ with $99.7 \%$ probability and the speed of sound values in the soft tissue are on the interval $[1530,1590] \mathrm{m} / \mathrm{s}$ with $99.7 \%$ probability.

In the computation of the forward solution, the computation domain was discretized into $300 \times 300$ with $33 \mu \mathrm{m}$ pixel width, and 4001 time steps at a temporal sampling rate of $333 \mathrm{MHz}$ were considered. The measured signal was downsampled to $66 \mathrm{MHz}$. In addition, a white Gaussian noise that had a zero mean and a standard deviation of $1 \%$ 


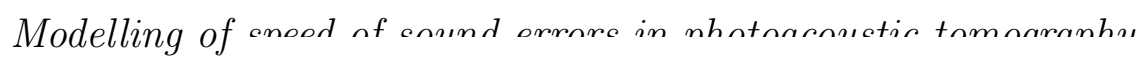
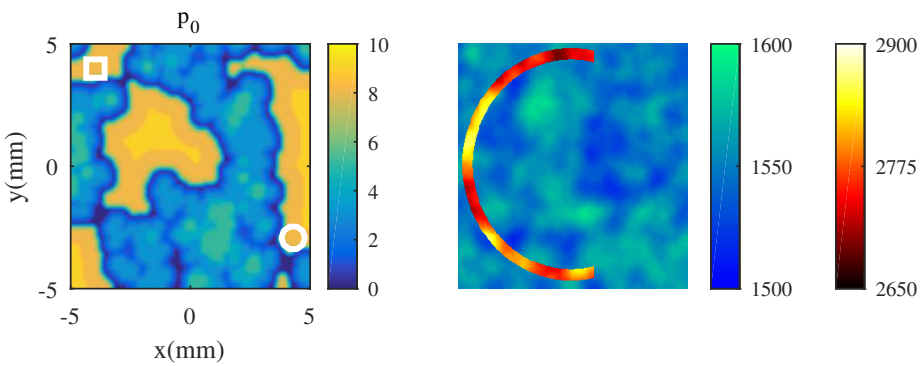

Figure 6. The simulated (true) initial pressure distribution $p_{0}$ and the speed of sound distribution $c_{3}$ used in the soft tissue and bone mimicking simulations. The initial pressure distribution is given in arbitrary units and the speed of sound distributions are given in units of $\mathrm{m} / \mathrm{s}$. The square and circle in the left image indicate the pixels where the covariances and marginal densities are plotted

Table 4. Parameters of the squared exponential distribution used in simulating the speed of sound distributions and in teaching the modelling error in the soft tissue and bone mimicking simulations: mean $\eta_{c}(\mathrm{~m} / \mathrm{s})$, standard deviation $\sigma_{c}(\mathrm{~m} / \mathrm{s})$ and characteristic length scale $l_{c}(\mathrm{~mm})$.

\begin{tabular}{llccc}
\hline & & $\eta_{c}$ & $\sigma_{c}$ & $l_{c}$ \\
\hline \multirow{2}{*}{ Data simulation } & Bone & 2760 & $170 / 6$ & 0.75 \\
& Tissue & 1560 & $60 / 6$ & 0.75 \\
\multirow{4}{*}{ Modelling error } & & & & \\
& Bone & 2750 & $300 / 6$ & 1 \\
& Tissue & 1550 & $100 / 6$ & 1 \\
\hline
\end{tabular}

of the maximum simulated data was added to these signals.

3.2.2. Approximation of the modelling error Statistics of the approximation error were estimated using 10000 initial pressure samples drawn from the Ornstein-Uhlenbeck distribution and 10000 speed of sound samples drawn from the squared exponential distribution. Parameters of the sample distributions are given in Table 5 for the initial pressure and in Table 4 for the speed of sound. In the initial pressure samples, negative values were not allowed and thus the absolute values of the samples were considered. Samples of the approximation error were computed from a set of forward solutions with the accurate and inaccurate models using (18). In the accurate model, the sampled speed of sounds were used. In the inaccurate model, the fixed constant value for the speed of sound was chosen to be $1500 \mathrm{~m} / \mathrm{s}$ for the soft tissue and $2750 \mathrm{~m} / \mathrm{s}$ for the bone, and boundaries of these regions were assumed to be known. The mean and the covariance of the modelling error were computed from approximation error samples using (19) and (20). 
Table 5. Parameters of the Ornstein-Uhlenbeck distribution used in simulating the initial pressure distributions when teaching the modelling error and in the solution of the inverse problem in the case of the tissue and bone mimicking simulations: mean $\eta_{p_{0}}$, standard deviation $\sigma_{p_{0}}$ and characteristic length scale $l_{p_{0}}(\mathrm{~mm})$.

\begin{tabular}{lccc}
\hline & $\eta_{p_{0}}$ & $\sigma_{p_{0}}$ & $l_{p_{0}}$ \\
\hline Modelling error & 5 & $10 / 6$ & 0.75 \\
Inverse problem & 5 & $10 / 2$ & 2 \\
\hline
\end{tabular}

3.2.3. Solution of the inverse problem In the computation of the inverse solution, the computation domain was discretized into $200 \times 200$ pixels with $50 \mu \mathrm{m}$ pixel width. The solution of inverse problem was computed using the accurate speed of sound (AFM), the inaccurate speed of sound (IFM), and the inaccurate speed of sound together with error modelling (IFM\&EM). In the case of the AFM, the true speed of sound distribution was interpolated from the simulation domain to the reconstruction domain. In the IFM and IFM\&EM, the fixed constant value for the speed of sound was chosen to be $1500 \mathrm{~m} / \mathrm{s}$ for the soft tissue and $2750 \mathrm{~m} / \mathrm{s}$ for the bone. In addition, the boundaries of the sub-regions were assumed to be known exactly. As prior information, the OrnsteinUhlenbeck process was used. Prior parameters are given in Table 5. Measurement noise was modelled as a Gaussian with zero mean and standard deviation set to $1 \%$ of the peak positive amplitude of the noisy simulated data.

3.2.4. Results The mean and standard deviation of the posterior distribution of the initial pressure obtained using different models and sensor geometries are shown in Figure 7. In addition, marginal densities at two pixels inside of the domain are shown in Figure 8. The relative errors of the estimates are given in Table 6.

As it can be seen, both the quality and the quantitative accuracy of the solution of the inverse problem depend on exactness of the used model. Accurate modelling of the speed of sound resulted in the most accurate estimates with the smallest relative errors. Use of an inaccurate piece-wise constant speed of sound in the solution inverse problem causes artefacts in the estimates of the posterior mean and results into significantly higher relative errors. Although the estimates of posterior mean are not equally accurate in the case of the true speed of sound and the inaccurate speed of sound, the standard deviations are similar. This indicates that the standard deviations are too small when the inaccurate speed of sound is used. On the other hand, accuracy and reliability of the solution of the inverse problem can be improved by modelling uncertainties in the speed of sound. Modelling of errors decreases the artefacts shown in the estimates of posterior mean and decreases relative errors, thus making these estimates comparable to the estimates with the accurate speed of sound. In addition, modelling of errors increases the standard deviations. For example, as it can be seen in Figure 8 the true value is within the principal support of the distribution in IFM\&EM estimate. 

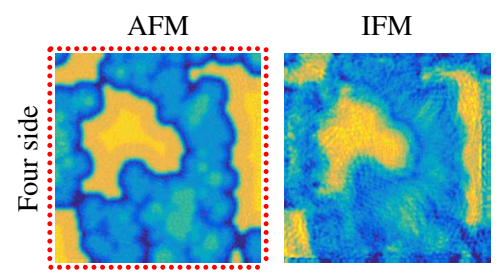

IFM\&EM
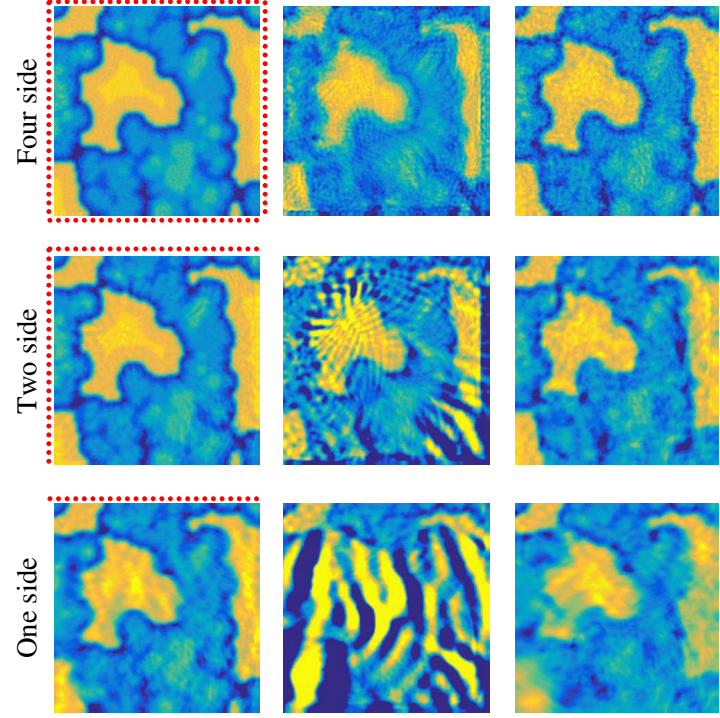

0

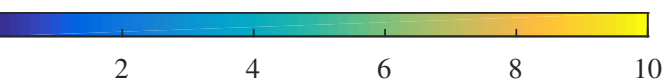

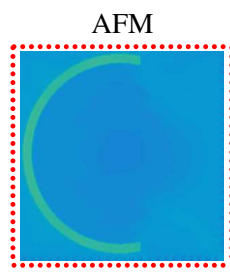

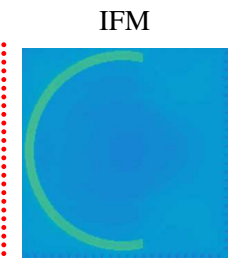

IFM\&EM
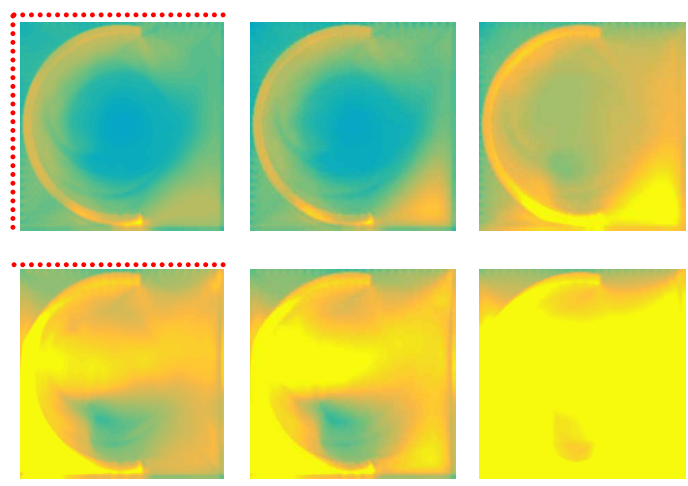

0

0.2

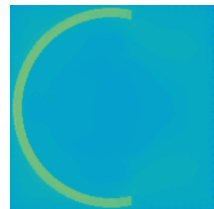

Figure 7. Posterior mean (left block) and standard deviation (right block) for the soft tissue and bone mimicking simulations. Shown are the results obtained using the AFM, IF) and IFM\&EM. The rows from top to bottom represent four side (first row), two side (second row) and one side (third row) sensor geometries. The red dots in the first column images indicate the locations of the sensors.

Table 6. Relative errors of the estimated mean of the posterior in percentage obtained using the AFM, IFM and IFM\&EM in four side, two sides and one side sensor geometries in the case of the soft tissue and bone mimicking simulations.

\begin{tabular}{lccc}
\hline & AFM & IFM & IFM\&EM \\
\hline Four side & 4 & 31 & 12 \\
Two side & 7 & 60 & 22 \\
One side & 15 & 129 & 33 \\
\hline
\end{tabular}

Comparing the estimates obtained using different sensor geometries, it can be seen that the most accurate estimates of the posterior mean are obtained using the full view sensor geometry and the accuracy of the estimates reduces in the limited view geometries. Correspondingly, the standard deviations are smallest in case of the full view sensor geometry and the use of the limited view sensor geometries increases the standard deviations. Reduction of the accuracy and increase in uncertainty is more significant in regions not enclosed by the sensors. This can be seen in Figure 8 where the maximum of marginal density is further from the true value and the true value is not within the principal support of the distribution. It also should be noted that the standard deviation is larger in the location on the bony structure than on the soft tissue, 

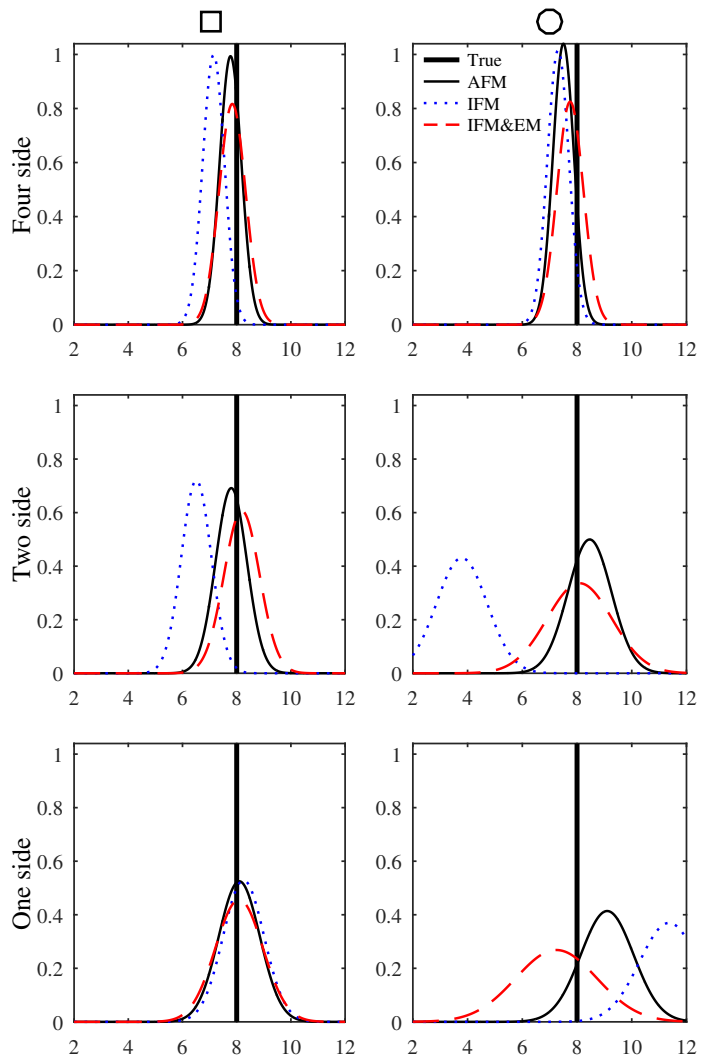

Figure 8. Marginal probability densities of the posterior distributions at locations denoted by $\square$ and $\bigcirc$ in Figure 6 for the soft tissue and bone mimicking simulations. The rows presents results obtained using four side (first row), two side (second row) and one side (third row) sensor geometries. Shown in the graphs are results for the AFM, IFM and IFM\&EM with black line denoting the true value initial pressure distribution.

and at least in the limited view sensor geometries, the bony structure also increases the standard deviation of the soft tissue near it. Furthermore, the bony structure distorts the standard deviation making the standard deviations not as smooth as in the case of the soft tissue mimicking simulations.

Covariance of the posterior distribution is visualized in Figure 9 by plotting covariance at two pixels inside of the domain. As it can be seen, the shapes of the covariance patterns are different compared to the soft tissue mimicking simulations. This likely arises from the bony structure. In addition, it can be noticed that modelling of the errors changes the covariance. This is more evident in the pixel $(\bigcirc)$ that is far from any sensor. Also, the sensor geometry changes the covariance. The correlation between the pixels increases and the covariance pattern change in shape as the number of the detection surfaces decreases. Especially, the pixel $(\bigcirc)$ that is distant from the sensor correlates more strongly with other pixels than the pixel $(\square)$ that is close to the sensor. 

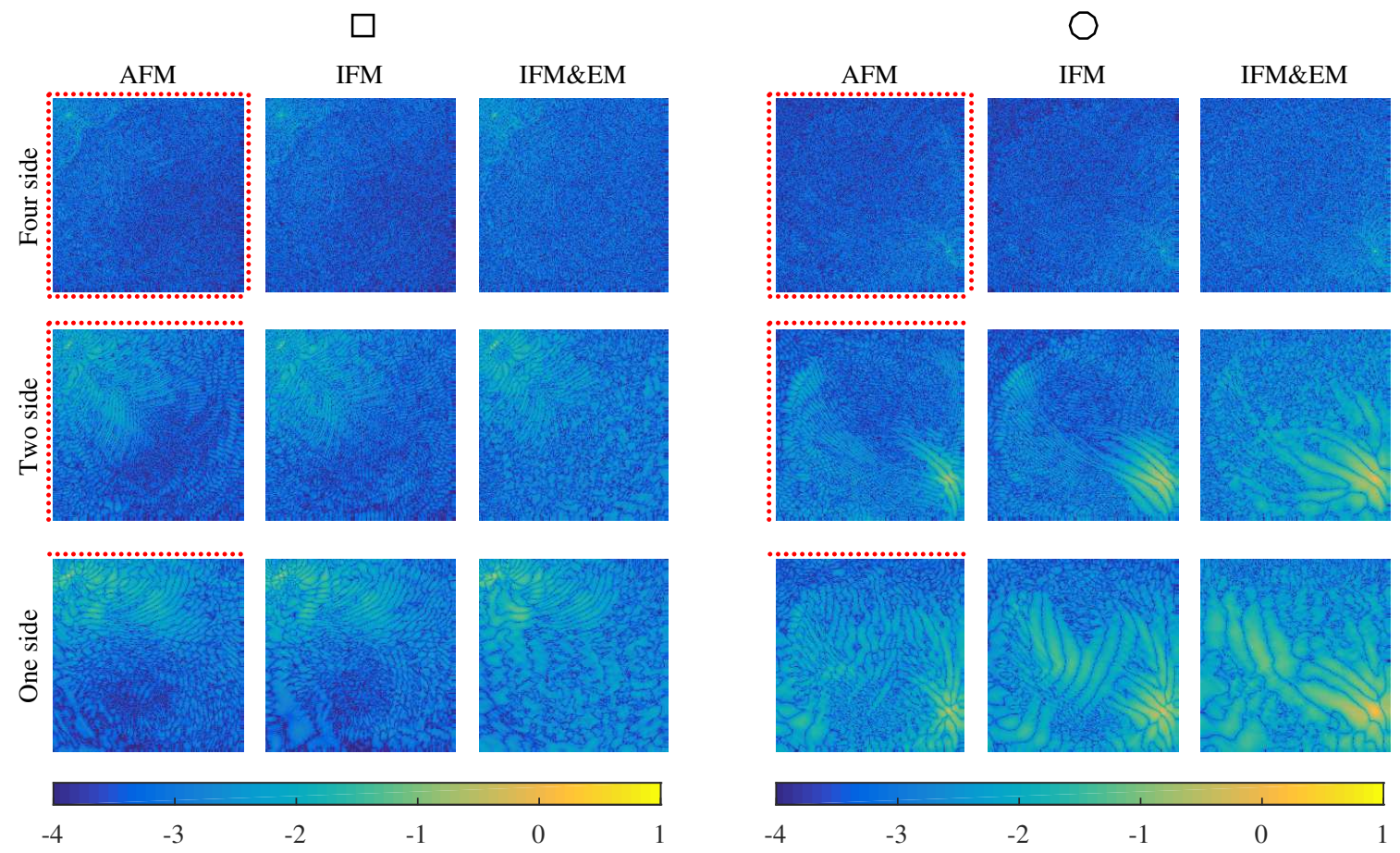

Figure 9. The absolute values of the posterior covariances of the initial pressure on a logarithmic scale at locations denoted by $\square$ and $\bigcirc$ in Figure 6 for the soft tissue and bone mimicking simulations. The left block presents the results in the point marked with $\square$ and the right block presents the results in the point marked with $\bigcirc$. Shown in the blocks are the results obtained using the AFM, IFM and IFM\&EM in four side (first row), two side (second row) and one side (third row) sensor geometry. The red dots in the first column images indicate the locations of the sensors.

\section{Discussion}

In this paper, the boundaries between soft tissue and a bone structure were assumed to be known. This boundary information could be obtained, for example, from a computed tomography (CT) image. However, these images are not always available. In addition, segmentation of the regions from these images can be difficult and thus errors may occur leading to errors in the estimates. Alternatively, it could be possible to utilize a similar reduced parametrization approaches as in [19] to find constant speed of sound values for the speed of sound in certain sub-regions, and then model the uncertainties using the approach proposed in this work. Furthermore, it could be possible to model the uncertainties related to the locations of the sub-region boundaries using the Bayesian approximation error modelling.

The performance of the approximation error modelling depends on the prior and teaching models that are used. The models should be chosen in such a way that the true target is well supported by the model. The choice of these models and their parameters is based on pre-existing knowledge of the imaged target. In practice, prior information on the speed of sound in various tissues exists as a tabulated data, and anatomical information could be obtained, for example, using an another imaging modality such as 
CT. Even though the prior model of the initial pressure utilized in this paper does not represent the simulated target exactly, the results show that accurate solutions of the inverse problem could still be achieved with this model. The results also suggests that the approximation error modelling is robust to errors in the assumed speed of sound used in the teaching of the approximation error. That is, even though the true speed of sound distribution is not fully covered by teaching distribution, the approximation error modelling can still correct some errors in the estimates.

In this paper, the medium was assumed to be non-attenuating and density variations were also ignored. However, a biological tissue has usually variations in the density values and it is attenuating. In future work, the approximation error modelling could be extended to compensate errors caused by the uncertainties in these parameters as well.

\section{Conclusions}

In this paper, errors caused by unknown speed of sound in PAT and modelling of these errors utilizing the Bayesian approximation error modelling were studied. The approach was evaluated with numerical simulations using different speed of sound distributions and sensor geometries. Posterior mean, standard deviations, covariances and marginal densities were inspected to evaluate the results.

Results show that inaccurate modelling of the speed of sound can lead to reduced accuracy of the estimates of the posterior mean and overly confident uncertainty estimates. However, modelling of the errors using the Bayesian approximation error modelling can improve the solution of the inverse problem significantly by improving the accuracy of the mean and making the credibilities more reliable. However, the Bayesian approximation error modelling is able to compensate only for small variations in the speed of sound.

\section{Acknowledgments}

This work has been supported by the Academy of Finland (projects 314411 and 312342 Centre of Excellence in Inverse Modelling and Imaging), Jane and Aatos Erkko Foundation, Saastamoinen Foundation and Alfred Kordelin foundation.

\section{References}

[1] Beard P 2011 Biomedical photoacoustic imaging. Interface Focus 1602

[2] Li C and Wang L V 2009 Photoacoustic tomography and sensing in biomedicine. Phys. Med. Biol. 54 R59

[3] Lutzweiler C and Razansky D 2013 Optoacoustic imaging and tomography: Reconstruction approaches and outstanding challenges in image performance and quantification. Sensors 13 7345

[4] Wang L V and Yao J 2016 A practical guide to photoacoustic tomography in the life sciences. Nat. Methods 13627 
[5] Xia J and Wang L V 2014 Small-animal whole-body photoacoustic tomography: a review. IEEE Trans. Biomed. Eng. 611380

[6] Xu M and Wang L V 2006 Photoacoustic imaging in biomedicine. Rev. Sci. Instrum. 77041101

[7] Zhou Y, Yao J and Wang L V 2016 Tutorial on photoacoustic tomography. J. Biomed. Opt. 21 061007

[8] Tick J, Pulkkinen A and Tarvainen T 2016 Image reconstruction with uncertainty quantification in photoacoustic tomography. J. Acoust. Soc. Am. 1391951

[9] Tick J, Pulkkinen A, Lucka F, Ellwood R, Cox B T, Kaipio J P, Arridge S R and Tarvainen T 2018 Three dimensional photoacoustic tomography in bayesian framework. J. Acoust. Soc. Am. 1442061

[10] Treeby B E, Varslot T K, Zhang E Z, Laufer J G and Beard P C 2011 Automatic sound speed selection in photoacoustic image reconstruction using an autofocus approach. J. Biomed. Opt. 16090501

[11] Anastasio M A, Zhang J, Pan X, Zou Y, Ku G and Wang L V 2005 Half-time image reconstruction in thermoacoustic tomography. IEEE Trans. Med. Imag. 24199

[12] Poudel J, Matthews T P, Li L, Anastasio M A and Wang L V 2017 Mitigation of artifacts due to isolated acoustic heterogeneities in photoacoustic computed tomography using a variable data truncation-based reconstruction method. J. Biomed. Opt. 22041018

[13] Zhang C and Wang Y 2008 A reconstruction algorithm for thermoacoustic tomography with compensation for acoustic speed heterogeneity. Phys. Med. Biol. 534971

[14] Deán-Ben X L, Ma R, Razansky D and Ntziachristos V 2011 Statistical approach for optoacoustic image reconstruction in the presence of strong acoustic heterogeneities. IEEE Trans. Med. Imag. 30401

[15] Huang C, Wang K, Schoonover R W, Wang L V and Anastasio M A 2016 Joint reconstruction of absorbed optical energy density and sound speed distributions in photoacoustic computed tomography: a numerical investigation. IEEE Trans. Comput. Imag. 2136

[16] Kirsch A and Scherzer O 2012 Simultaneous reconstructions of absorption density and wave speed with photoacoustic measurements. SIAM J. Appl. Math 721508

[17] Liu H and Uhlmann G 2015 Determining both sound speed and internal source in thermo-and photo-acoustic tomography. Inverse Probl. 31105005

[18] Yuan Z, Zhang Q and Jiang H 2006 Simultaneous reconstruction of acoustic and optical properties of heterogeneous media by quantitative photoacoustic tomography. Opt. Express 146749

[19] Matthews T P, Poudel J, Li L, Wang L V and Anastasio M A 2018 Parameterized joint reconstruction of the initial pressure and sound speed distributions for photoacoustic computed tomography. SIAM J. Imaging Sci. 111560

[20] Stefanov P and Uhlmann G 2013 Instability of the linearized problem in multiwave tomography of recovery both the source and the speed. Inverse Probl. Imag. 7

[21] Jose J, Willemink R G, Steenbergen W, Slump C H, van Leeuwen T G and Manohar S 2012 Speed-of-sound compensated photoacoustic tomography for accurate imaging. Med. Phys. 39 7262

[22] Manohar S, Willemink R G, van der Heijden F, Slump C H and van Leeuwen T G 2007 Concomitant speed-of-sound tomography in photoacoustic imaging. Appl. Phys. Lett. 91131911

[23] Matthews T P and Anastasio M A 2017 Joint reconstruction of the initial pressure and speed of sound distributions from combined photoacoustic and ultrasound tomography measurements. Inverse Probl. 33124002

[24] Xia J, Huang C, Maslov K, Anastasio M A and Wang L V 2013 Enhancement of photoacoustic tomography by ultrasonic computed tomography based on optical excitation of elements of a full-ring transducer array. Opt. Lett. 383140

[25] Kaipio J and Somersalo E 2006 Statistical and Computational Inverse Problems (Springer Science \& Business Media)

[26] Tarantola A 2005 Inverse Problem Theory and Methods for Model Parameter Estimation (Society 
for Industrial and Applied Mathematics)

[27] Pulkkinen A, Kolehmainen V, Kaipio J P, Cox B T, Arridge S R and Tarvainen T 2014 Approximate marginalization of unknown scattering in quantitative photoacoustic tomography. Inverse Probl. Imag. 8811

[28] Tarvainen T, Pulkkinen A, Cox B T, Kaipio J P and Arridge S R 2013 Bayesian image reconstruction in quantitative photoacoustic tomography. IEEE Trans. Med. Imag. 322287

[29] Hänninen N, Pulkkinen A and Tarvainen T 2018 Image reconstruction with reliability assessment in quantitative photoacoustic tomography. J. Imaging 4148

[30] Arridge S R, Kaipio J P, Kolehmainen V, Schweiger M, Somersalo E, Tarvainen T and Vauhkonen M 2006 Approximation errors and model reduction with an application in optical diffusion tomography. Inverse Probl. 22175

[31] Kolehmainen V, Schweiger M, Nissilä I, Tarvainen T, Arridge S R and Kaipio J P 2009 Approximation errors and model reduction in three-dimensional diffuse optical tomography. J. Opt. Soc. Am. A 262257

[32] Tarvainen T, Kolehmainen V, Pulkkinen A, Vauhkonen M, Schweiger M, Arridge S R and Kaipio J P An approximation error approach for compensating for modelling errors between the radiative transfer equation and the diffusion approximation in diffuse optical tomography. Inverse Probl. 26

[33] Tarvainen T, Kolehmainen V, Kaipio J P and Arridge S R 2010 Corrections to linear methods for diffuse optical tomography using approximation error modelling. Biomed. Opt. Express 1209

[34] Mozumder M, Tarvainen T, Arridge S R, Kaipio J P and Kolehmainen V 2013 Compensation of optode sensitivity and position errors in diffuse optical tomography using the approximation error approach. Biomed. Opt. Express 42015

[35] Mozumder M, Tarvainen T, Kaipio J P, Arridge S R and Kolehmainen V 2014 Compensation of modeling errors due to unknown domain boundary in diffuse optical tomography. J. Opt. Soc. Am. A 311847

[36] Heino J, Somersalo E and Kaipio J P 2005 Compensation for geometric mismodelling by anisotropies in optical tomography. Opt. Express 13296

[37] Koponen J, Huttunen T, Tarvainen T and Kaipio J P 2014 Bayesian approximation error approach in full-wave ultrasound tomography. IEEE Trans. Ultrason., Ferroelectr., Freq. Control 611627

[38] Treeby B E and Cox B T 2010 k-wave: Matlab toolbox for the simulation and reconstruction of photoacoustic wave fields. J. Biomed. Opt. 15021314

[39] Koulouri A, Rimpilinen V, Brookes M and Kaipio J P 2016 Compensation of domain modelling errors in the inverse source problem of the poisson equation: Application in electroencephalographic imaging. Appl. Numer. Math. 10624

[40] Rasmussen C E and Williams C K I 2006 Gaussian Processes for Machine Learning (MIT Press) 